\title{
Calcium determination in biochar-based fertilizers by laser-induced breakdown spectroscopy using sodium as internal standard
}

\author{
Carla Pereira de Morais a , Ariane Isis Barros a , Dário Santos Júnior ${ }^{\mathrm{b}}$, Clóvis Augusto Ribeiro a \\ Marisa Spirandeli Crespi ${ }^{\mathrm{a}}$, Giorgio Saverio Senesi ${ }^{\mathrm{c}}$, José Anchieta Gomes Neto ${ }^{\mathrm{a}}$, Edilene Cristina Ferreira ${ }^{\mathrm{a}, *}$ \\ a São Paulo State University - UNESP, Analytical Chemistry Department, P.O. Box 355, 14801-970 Araraquara, SP, Brazil \\ ${ }^{\mathrm{b}}$ Federal University of São Paulo, UNIFESP, Chemistry Department, Rua São Nicolau, $n^{\circ} 210$ - Centro - CEP: 09913-030, Diadema, SP, Brazil \\ c CNR, NANOTEC - PlasMI Lab, Via Amendola 122/D, 70126 Bari, Italy
}

\section{A R T I C L E I N F O}

\section{Article history:}

Received 19 April 2017

Received in revised form 5 July 2017

Accepted 5 July 2017

Available online 6 July 2017

\section{Keywords:}

Biochar-based fertilizers

Calcium

Libs

Internal standardization

\begin{abstract}
A B S T R A C T
Biochar has gained agricultural importance as a soil amendment because of its important agricultural properties such as water retention, plant nutrient supplier, promoter of microorganism growth, sequestration action of atmospheric $\mathrm{CO}_{2}$, etc. Further, it is a low cost material being produced by recycling. Due to its active sites, biochar can adsorb nutrients so acting as a soil fertilizer. Thus the rapid assessment of nutrients in these materials is essential to ensure quality control for agricultural purposes. This work aimed to develop a simple analytical method based on Laser-Induced Breakdown Spectroscopy (LIBS) to determine Ca in biochar-based fertilizers. In particular, biochar samples enriched with Ca were prepared from peanut shells, residues of eucalyptus and banana fibers. The calibration standards were prepared by matrix matching using a biochar from eucalyptus residues. Different spectral preprocessing were evaluated to enhance the precision and accuracy of the method. However, the matrix effects demanded the use of internal standardization as the appropriate methodology to obtain the best accuracy. A linear correlation coefficient of 0.989 and a linear work range of $1.51-11.23 \%$ Ca were obtained using the proposed method, which yielded limits of detection and quantification of $0.45 \%$ e $1.51 \%$, respectively. Calcium contents determined by LIBS in biochar-based fertilizers were in good agreement (paired $t$-test at 95\% confidence level) with those determined by using High-Resolution Continuous Source Atomic Absorption Spectrometry (HR-CS FAAS) as the reference technique. Thus, the importance of internal standardization was demonstrated to be successful for the quantitative analysis of $\mathrm{Ca}$ in complex matrices like biochar-based fertilizers.
\end{abstract}

(c) 2017 Elsevier B.V. All rights reserved.

\section{Introduction}

The use of biochar in agriculture to improve soil quality is a millenarian practice used by indigenous tribes of the central Amazonian basin, which resulted in a kind of soil known as indian dark earth [1]. The high fertility of Amazon Indians soils has been attracted the interest of soil scientists due to the intrinsic low fertility of tropical Amazonian soils are originally low fertility [2].

Currently this practice is known as pyrolytic conversion of agricultural waste into biochar, which is considered a soil enhancer due to its capacity to hold carbon, to assist water and nutrients retention and increase soil biodiversity $[3,4]$. The interest in biochar is growing up, driven by two major global issues, i.e. climate change and need for a more sustainable soil management [1]. In this context, Dias et al. [5] have recently proposed the use of biochar as potential fertilizer, possibly working by releasing nutrients in a controlled manner. In face of the

\footnotetext{
* Corresponding author.

E-mail address: edilene@iq.unesp.br (E.C. Ferreira).
}

perspective of biochar use for agricultural purposes, the International Biochar Initiative (IBI), started in 2006 during the World Soil Science Congress (WSSC), has highlighted the importance of the analytical characterization and producer's certification of biochar used as soil amendment [6].

The consolidated analytical techniques commonly used for elemental determinations require the dissolution of the solid sample, which involves the use of strong oxidants and high temperatures [7,8]. However, biochar consists of a recalcitrant material that is hardly soluble under normal conditions of wet decomposition, thus requiring more drastic conditions, such as high pressures or decomposition in dry conditions using fluxing agents [9]. As consequence, the use of the aforementioned conditions increases the possibility of systematic errors besides producing large volumes of chemical residues [10].

Laser-Induced Breakdown Spectroscopy (LIBS) is an analytical technique able to perform direct simultaneous multielemental analysis of solid samples by applying a highly energetic laser pulse on the sample surface, so promoting the sample breakdown, plasma generation and species excitation in a single step $[11,12]$. Then, the characteristic 
wavelengths of the radiation emitted by de-excitation of the species in the plasma are recorded simultaneously, so yielding a sample emission spectrum that provides a qualitative overview of sample composition. The quantitative information is then extracted from the emission signal magnitudes, which are related to standard concentrations through a suitable calibration model [11,13-15]. Linear calibration models are more adequate, considering the emission intensities of excited elements are directly proportional to their concentration [16]. In particular, due to the common fluctuations observed in LIBS measurements associated to both, instrumentation and sample heterogeneity, various strategies are experimented for the calibration of LIBS methods, which include various different spectral preprocessing and multivariate (linear and non-linear) calibration models [17-20]. Among these, internal standardization, which consists in normalizing the analyte signal by the signal of an internal standard (IS), is a well known methodology used to minimize fluctuations in spectroscopic techniques including LIBS [21-24]. To perform internal standardization, the IS concentration must be known and nearly constant [25,26]. However, in some cases it is difficult to select an adequate IS in LIBS analysis because the IS may be subject to temporal processes different from those experienced by the analyte in the plasma. The use as IS of an element naturally present in the samples analyzed may also be inappropriate as the IS concentration may change from sample to sample, thus not fitting the IS requirements $[22,23,27]$.

In this work, different spectral preprocessing and internal standardization methods were evaluated to find out the best methodology that can allow minimizing fluctuations in LIBS measurements, so enabling the adequate LIBS analysis of Ca in biochar-based fertilizers.

\section{Materials and methods}

\subsection{Eucalyptus biochar production}

The original eucalyptus biochar was produced by using leaves and branches of eucalyptus. The materials were mixed and crushed using a knife mill from Tecnal (Willey TE 340, Piracicaba, Brazil). Then $2.0 \mathrm{~g}$ of this powdered biomass were transferred to a porcelain crucible capped with aluminum paper to keep the atmosphere rare in oxygen, heated in a muffle oven from EDG (EDGCON 5P, São Carlos, Brazil) from room temperature to $260{ }^{\circ} \mathrm{C}$ at a heating rate of $10^{\circ} \mathrm{C} \mathrm{min}^{-1}$, and finally maintained at $260^{\circ} \mathrm{C}$ for $60 \mathrm{~min}$. After cooling to room temperature, the biochar was stored in an amber flask inside a desiccator to be used in standards calibration for matrix matching.

\subsection{Fertilizer samples production}

Fertilizer samples were produced from leaves and branches of eucalyptus, banana fibers and peanut shells. In particular, $3.0 \mathrm{~g}$ of each biomass were placed in Erlenmeyer flasks containing $100 \mathrm{~mL}$ of $1.80 \mathrm{~g} \mathrm{~L}^{-1} \mathrm{Ca}^{2+}$ water solution prepared from CaO (Alfa Aesar), and then shaken at $25^{\circ} \mathrm{C}$ for $24 \mathrm{~h}$ in a incubator with orbital agitation at $150 \mathrm{rpm}$. After filtration, the biomasses were dried at $40^{\circ} \mathrm{C}$ for $24 \mathrm{~h}$. Then $3.0 \mathrm{~g}$ of each dried biomass were submitted to the same procedure and heating program described above to produce the eucalyptus biochar. Each fertilizer was prepared in triplicate.

An aliquot of $200 \mathrm{mg}$ of each fertilizer so produced was mixed with $80 \mathrm{mg}$ of cellulose (type 101 Sigmacell) for sample compaction, homogenized in an agate mortar, and finally converted into pellets by applying a pressure of 10 tons in a hydraulic press from Specac (GS15011, Swedesboro, USA).

\subsection{LIBS analysis}

The LIBS instrument used was a homemade system equipped with a Q-switched Nd:YAG laser from Quantel (Big Sky Ultra 50, Bozeman, USA) emitting at $1064 \mathrm{~nm}$ and operating at a maximum power energy of $50 \mathrm{~mJ}$ and a repetition rate of up to $20 \mathrm{~Hz}$, with a pulse duration of
$20 \mathrm{~ns}$, and a laser spot diameter of about $0.5 \mathrm{~mm}$. The detection device used was the spectrometer from Ocean Optics (HR2000+, Dunedin,USA) that is composed of four spectrometers with optical resolution of 0.1-0.2 nm (FWHM) which measure the range between 199 and $631 \mathrm{~nm}$ at a fixed integration time of $1 \mu$ s. Individual samples were placed in an electronically controlled sampling chamber with movement in the $x-y$ directions and equipped with a video camera for monitoring the process. The Q-Switched delay time selected for spectra acquisition was $2.0 \mu \mathrm{s}$. The system was controlled by the OOLIBS software (Ocean Optics). Sixty LIBS spectra were acquired from each standard and sample pellet. The average spectra of individual studied biomasses plus cellulose are shown in the Supplementary Material (Fig. S1).

The application of a univariate linear calibration model was evaluated by the Least-Squares Regression method for the Ca atomic emission line at $612.22 \mathrm{~nm}$. This low relative intensity emission line (13\%) was selected to avoid the self-absorption effects that were observed for more sensitive lines. The intensity of the peak, the peak area fitted by the Lorentz and Voigt functions and the maximum variation ratio of the first derived spectrum were evaluated in spectral preprocessing.

The calibration standards were prepared by matrix matching by mixing $156 \mathrm{mg}$ of eucalyptus biochar, $80 \mathrm{mg}$ of cellulose, $\mathrm{CaO}$ at different amounts to produce Ca standards with the following concentrations: $0,1.02,2.55,5.10,8.17$ and $11.23 \%(w / w)$, and maize starch in different amounts up to $280 \mathrm{mg}$. All components were homogenized using an agate mortar and pistil, and then compacted in pellets using a pressure of 10 tons. For calibration purposes internal standardization was also evaluated by using as IS the Na atomic line at $588.99 \mathrm{~nm}$ to normalize the $\mathrm{Ca}$ atomic line after spectrum derivation.

\subsection{Thermal and atomic absorption spectrometry analyses}

Thermal analysis was performed on the original eucalyptus biochar, the eucalyptus fertilizer sample and a calibration standard by simultaneous TGA-DTA using a TA Instruments apparatus (SDT 2960, New Castle, USA). Samples were heated from room temperature up to $800^{\circ} \mathrm{C}$ at a heating rate of $30{ }^{\circ} \mathrm{C} \mathrm{min}^{-1}$ under compressed air atmosphere and a flow rate of $100 \mathrm{~mL} \mathrm{~min}^{-1}$.

In order to check the accuracy of the LIBS method, the Ca contents in fertilizer samples were determined by High Resolution Continuous Source Flame Atomic Absorption Spectrometry (HR-CS FAAS) from AnalytikJena (ContrAA 300 spectrometer, Jena, Germany). The instrumental conditions used for analysis were: flame of nitrous oxide and acetylene at a flowing rate of 210 and $80 \mathrm{~L} \mathrm{~min}^{-1}$, respectively, burner $(50 \mathrm{~mm})$ at a height of $6 \mathrm{~mm}$, and aspiration rate of $5 \mathrm{~mL} \mathrm{~min}^{-1}$. The Ca line monitored was at $239.8559 \mathrm{~nm}$ (secondary line, with $1.1 \%$ of sensitivity).

The HR-CS FAAS analysis was conducted on sample digests prepared as follows. Fertilizers samples were mineralized in triplicate in a closevessels microwave-assisted acid-digestion system. Specifically, $100 \mathrm{mg}$ of each sample were accurately weighed and transferred to a microwave flask, to which $3.0 \mathrm{~mL}$ of $70 \%(\mathrm{v} / \mathrm{v})$ nitric acid solution, $2.0 \mathrm{~mL}$ of deionized water and $1.0 \mathrm{~mL}$ of $\mathrm{H}_{2} \mathrm{O}_{2} 30 \%(\mathrm{v} / \mathrm{v})$ were added. The mixtures were heated in a microwave oven from Anton Paar (Multiwave, Graz, Austria) using an optimized program involving power/ramp time/ hold time of $900 \mathrm{~W} / 15 \mathrm{~min} / 30 \mathrm{~min}$, respectively. After digesting and cooling, the resulting digests were transferred to $25 \mathrm{~mL}$ volumetric flasks, and the volume completed with de-ionized water. The accuracy of the reference method was evaluated using the certified reference material Pine Needles (NIST - 1575a).

\section{Results and discussions}

Calibration models were calculated for the direct correlation between Ca concentration and parameters extracted from the LIBS emission signal of Ca I $612.22 \mathrm{~nm}$ in calibration standards. To this purpose, 


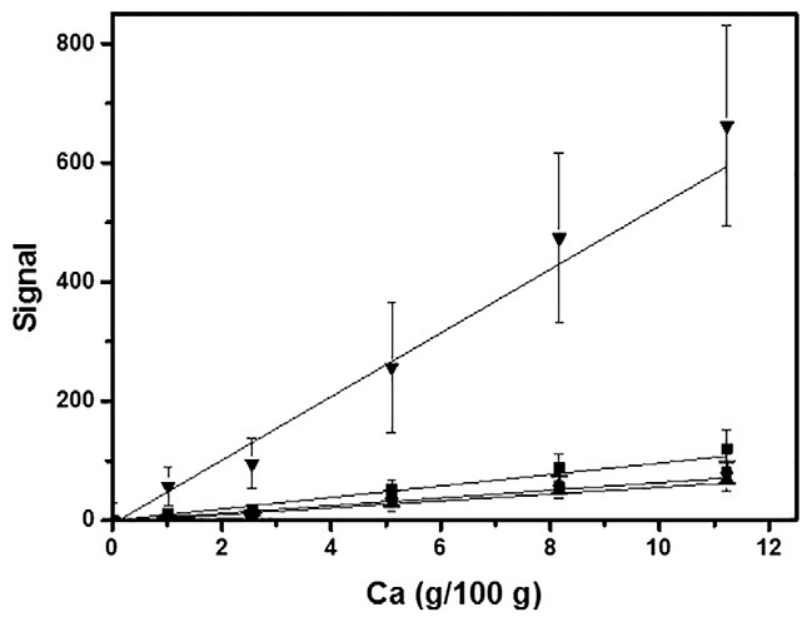

Fig. 1. Evaluated calibration curves for Ca determination based on: peak intensity ( $\mathbf{\square})$, peak area fitted by Lorentz function $(\boldsymbol{\bullet})$, peak area fitted by Voigt function $(\boldsymbol{\Delta})$ and maximum variation rate of derived signal $(\boldsymbol{\nabla})$.

LIBS spectra were preprocessed in different ways, aiming to minimize common fluctuations in measurements and obtain a suitable calibration model. The four evaluated models considered (Fig. 1), based on the spectral information of peak intensity, peak area fitted by Lorentz function, peak area fitted by Voigt function and maximum variation rate of derived signal, showed correlation coefficients of $0.974,0.963,0.952$ and 0.971 , respectively. Additionally, the amount of $\mathrm{Ca}(\mathrm{g} / 100 \mathrm{~g})$ predicted by these models were $1.20 \pm 0.35 \%, 1.20 \pm 0.08 \%, 1.68 \pm$ $0.20 \%, 1.29 \pm 0.11 \%$, respectively. These results indicated that well fitted analytical curves with similar prediction capabilities were obtained. Further, the application of peak areas or spectrum differentiation caused a considerable reduction of the relative standard deviation that dropped from $29 \%$ obtained by the peak intensity method to an average of $9 \%$. However, the prediction accuracy was not acceptable because the $\mathrm{Ca}$ concentration determined in the sample by the reference method was

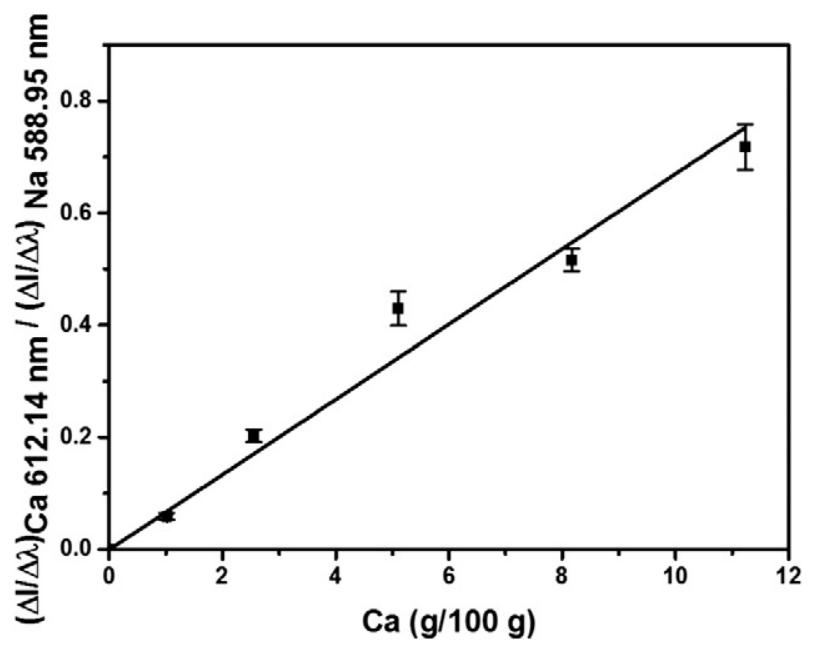

Fig. 3. Calibration curve for Ca determination by LIBS using Na as internal standard.

$5.38 \pm 0.27 \%$. These results suggested that, although fluctuations were minimized by spectral preprocessing, the matrix effect persisted causing an underestimation of the Ca content in the sample.

To evaluate the occurrence of matrix effects, thermal analysis was performed on the original biochar, the biochar-based fertilizer and a calibration standard (Fig. 2). The DTA curves show that the sum of the events is exothermic, and the TG and DTG curves indicate that the three events imply a weight loss. The first event can be ascribed to sample dehydration, the second one to evaporation of volatile compounds, and the third to the decomposition of $\mathrm{CaCO}_{3}$ in $\mathrm{CaO}(\mathrm{s})$ and $\mathrm{CO}_{2}(\mathrm{~g})$ [28]. The analysis of the third event, in which $\mathrm{Ca}$ is involved, shows that: for the original biochar (Fig. 2a) the event started at $572.80{ }^{\circ} \mathrm{C}$ and was completed at $770.97^{\circ} \mathrm{C}$ with $1.43 \%$ of weight loss; for the fertilizer (Fig. 2b) the event started at $597.57{ }^{\circ} \mathrm{C}$ and finished at $771.86{ }^{\circ} \mathrm{C}$ with $8.20 \%$ of weight loss; and for the calibration standard (Fig. 2c) the event started at $559.01{ }^{\circ} \mathrm{C}$ and finished at $773.82{ }^{\circ} \mathrm{C}$ with $5.83 \%$ of
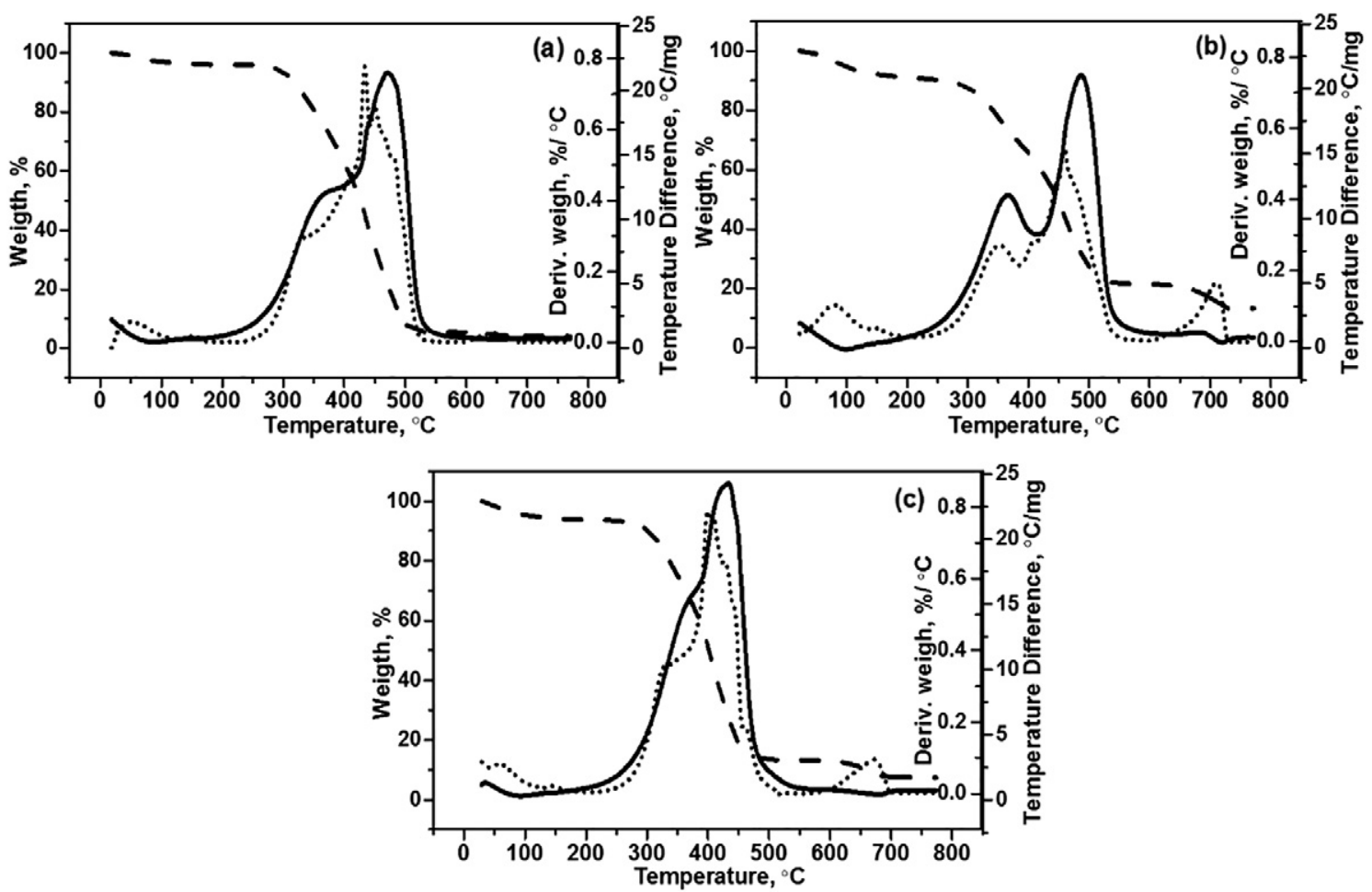

Fig. 2. TG (- - ), DTG (…) e a DTA (-) curves of (a) eucalyptus biochar, (b) eucalyptus fertilizer biochar, and (c) calibration standard containing 5.19 Ca\%. 
weight loss. These results would suggest that the fertilizer and the calibration standard are more similar compared to the original biochar, thus suggesting that $\mathrm{Ca}$ incorporation changes the original biochar properties. Thus, the use of matrix matching for Ca determination seems to be a good choice.

Despite the similarity between the standard and fertilizer sample, the different weight loss measured suggests that Ca was incorporated in a different way in the two materials. The original biochar possesses many active sites available for Ca incorporation by simple physical mixing [29], whereas incorporation would occur differently during the pyrolysis of Ca-added biochar. The different way in which elements are bound to the matrix probably causes the standard and the samples to experience differentiated breakdown and excitation processes during LIBS analysis.

In the attempt to minimize the differences between the sample and the calibration standard, the internal standardization method was also applied. The LIBS spectrum of the original eucalyptus biochar shows an unresolved line in the range of 585 to $595 \mathrm{~nm}$ that corresponds to a doublet of $\mathrm{Na}$ atomic lines ( 588.99 and $589.59 \mathrm{~nm}$ ). Considering that $\mathrm{Na}$ is from the biochar and this matrix is similarly diluted in samples and standards, a similar Na concentration is expected in both materials. Thus, Na was used as IS for Ca determination.

To minimize fluctuations the spectrum derivation method was used in spectral preprocessing. A well fitted linear correlation $(R=0.989)$ was obtained by plotting the ratio of the maximum variation rate of Ca I $612.22 \mathrm{~nm}$ to the maximum variation rate of $\mathrm{Na}$ I $588.99 \mathrm{~nm}$ as a function of the $\mathrm{Ca} \%$ in the calibration standard (Fig. 3). This calibration model was applied for Ca concentration predictions by LIBS in the biochar-based fertilizers. The biochar fertilizers of eucalyptus, peanut shells and banana fiber showed Ca\% of $5.89 \pm 0.70,3.69 \pm 0.56$ and $8.71 \pm$ 1.13 , respectively. These values are in good agreement (paired $t$-test at a $95 \%$ confidence level) with reference values, $5.38 \pm 0.27,3.71 \pm$ $0.22,9.17 \pm 0.13$, respectively. Further, the LIBS method featured a linear work range of $1.51-11.23 \% \mathrm{Ca}$, and limits of detection and quantification of $0.45 \%$ and $1.51 \%$ respectively, as calculated according to Currie [30].

\section{Conclusions}

The application of the LIBS technique to the determination of Ca in biochars-based fertilizers showed the need of a preliminary spectral processing in order to minimize the common fluctuations occurring in LIBS measurements, which usually decrease their precision. Due to the occurrence of matrix effects, as proven by thermal analysis, the prediction accuracy of the method could not be achieved by applying common spectral preprocessing methods, but using the internal standardization method based on Na naturally present in the samples, which permitted the proper correction of the Ca signal.

The LIBS method developed appeared to respond to the IBI demands as it shows promising analytical features, i.e. it is fast, does not require drastic preparation conditions and is environmentally friend because it does not generate chemical residues of analysis. Further, LIBS can be adapted to other types of coals of difficult decomposition.

Supplementary data to this article can be found online at http://dx. doi.org/10.1016/j.microc.2017.07.005.

\section{Acknowledgements}

The authors thank the Fundação de Amparo à Pesquisa do Estado de São Paulo (FAPESP) for financial support of this work (Grant \# 2015/ 24757-9), the Coordenação de Aperfeiçoamento Pessoal de Nível Superior (CAPES) for providing a fellowship to C.P.M, and the Conselho Nacional de Desenvolvimento Científico e Tecnológico for a fellowship to A.I $\cdot$ B and a research grant to J.A.G.N and D.S.J.

\section{References}

[1] K.Y. Chan, L. van Zwieten, I. Meszaros, A. Downie, S. Joseph, Agronomic values of greenwaste biochar as a soil amendment, Aust. J. Soil Res. 45 (2007) 629-634.

[2] B. Glaser, L. Haumaier, G. Guggenberger, W. Zech, The "Terra Preta" phenomenon: a model for sustainable agriculture in the humid tropics, Naturwissenschaften 88 (2001) 37-41.

[3] C.J. Atkinson, J.D. Fitzgerald, N.A. Hipps, Potential mechanisms for achieving agricultural benefits from biochar application to temperate soils: a review, Plant Soil 337 (2010) 1-18.

[4] Y. Fang, B. Singh, B.P. Singh, E. Krull, Biochar carbon stability in four contrasting soils, Eur. J. Soil Sci. 65 (2014) 60-71.

[5] D.S. Dias, M.S. Crespi, L.D.M. Torquato, M. Kobelnik, C.A. Ribeiro, Torrefied banana tree fiber pellets having embedded urea for agricultural use, J. Therm. Anal. Calorim. (2017) 1-8.

[6] International Biochar Initiative (IBI), http://www.biochar-international.org 2017 (accessed 04.18.17).

[7] H.M. Kingston, S.J. Haswell, Microwave-Enhanced Chemistry: Fundamentals, Sample Preparation, and Applications, first ed. American Chemical Society, Washington, DC, 1997.

[8] US Environmental Protection Agency (EPA), Microwave-assisted acid digestion of siliceous and organically based matrices, method 3052, Office of Solid Waste and Emergency Response, U.S. Government Printing Office, Washington, D.C, 1996.

[9] G. Corazza, A.S. Henn, M.F. Mesko, F.A. Duarte, E.M.M. Flores, P.A. Mello, Microwaveinduced combustion of coal for further sulfur determination by inductively coupled plasma optical emission spectrometry or ion chromatography, J. Braz. Chem. Soc. 27 (2016) 1569-1576.

[10] P.T. Anastas, Green chemistry and the role of analytical methodology development, Crit. Rev. Anal. Chem. 29 (1999) 167-175.

[11] A.W. Miziolek, V. Palleschi, I. Schechter, Laser-Induced Breakdown Spectroscopy (LIBS): Fundamentals and Applications, Cambridge University Press, New York, 2006.

[12] D.W. Hahn, N. Omenetto, Laser-induced breakdown spectroscopy (LIBS), part I: review of basic diagnostics and plasmaparticle interactions: still-challenging issues within the analytical plasma community, Appl. Spectrosc. 64 (2010) 335A-366A.

[13] A.P.M. Michel, Review: applications of single-shot laser-induced breakdown spectroscopy, Spectrochim, Acta Part B 65 (2010) 185-191.

[14] L. Radziemski, D. Cremers, A brief history of laser-induced breakdown spectroscopy: from the concept of atoms to LIBS 2012, Spectrochim. Acta B 87 (2013) 3-10.

[15] P. Pořízka, A. Demidov, J. Kaiser, J. Keivanian, I. Gornushkin, U. Panne, J. Riedel, Laserinduced breakdown spectroscopy for in situ qualitative and quantitative analysis of mineral ores, Spectrochim. Acta B 101 (2014) 155-163.

[16] D.A. Skoog, F.J. Holler, S.R. Crouch, Principles of Instrumental Analysis, sixth ed. Brooks/Cole, Boston, 2006.

[17] E.C. Ferreira, D.M.B.P. Milori, E.J. Ferreira, R.M. Da Silva, L. Martin-Neto, Artificial neural network for Cu quantitative determination in soil using a portable laser induced breakdown spectroscopy system, Spectrochim. Acta B 63 (2008) 1216-1220.

[18] D. Santos, L.C. Nunes, G.G.A. De Carvalho, M.D.S. Gomes, P.F. de Souza, F.D.O. Leme, L.G.C. dos Santos, F.J. Krug, Laser-induced breakdown spectroscopy for analysis of plant materials: a review, Spectrochim. Acta B 71-72 (2012) 3-13.

[19] D.A. Cremers, L.J. Radziemski, Handbook of Laser-Induced Breakdown Spectroscopy, John Wiley \& Sons, West Sussex, 2013.

[20] J. El Haddad, L. Canioni, B. Bousquet, Good practices in LIBS analysis: review and advices, Spectrochim. Acta B 101 (2014) 171-182.

[21] K.G. Fernandes, M. de Moraes, J.A. Gomes Neto, J.A. Nóbrega, P.V. Oliveira, Padronização interna em espectrometria de absorção atômica, Quim Nova 26 (2003) 249-252

[22] S. Yao, J. Xu, K. Bai, J. Lu, Improved measurement performance of inorganic elements in coal by laser-induced breakdown spectroscopy coupled with internal standardization, Plasma Sci. Technol. 17 (2015) 938-943.

[23] H.K. Sanghapi, K.K. Ayyalasomayajula, F.Y. Yueh, J.P. Singh, D.L. McIntyre, J.C. Jain, J. Nakano, Analysis of slags using laser-induced breakdown spectroscopy, Spectrochim. Acta B 115 (2016) 40-45.

[24] H. Shirvani-Mahdavi, P. Shafiee, Quantitative analysis of soil calcium by laser-induced breakdown spectroscopy using addition and addition-internal standardizations, Meas. Sci. Technol. 27 (2016).

[25] W. Gerlach, E. Schweitezer, Foundations and Methods of Chemical Analysis by Emission Spectrometry, Adam Hilger, London, 1929.

[26] M.A. Bechlin, E.C. Ferreira, J.A. Gomes Neto, J.C. Ramos, D.L.G. Borges, Contributions on the use of bismuth as internal standard for lead determinations using ICPbased techniques, J. Braz. Chem. Soc. 26 (2015) 1879-1886.

[27] L. Sheng, T. Zhang, K. Wang, H. Tang, H. Li, Quantitative analysis of Fe content in iron ore via external calibration in conjunction with internal standardization method coupled with LIBS, Chem. Res. Chin. Univ. 31 (2014) 107-111.

[28] R.L. Frost, M.C. Hales, W.N. Martens, Thermogravimetric analysis of selected group (II) carbonateminerals - implication for the geosequestration of greenhouse gases, J. Therm. Anal. Calorim. 95 (2009) 999-1005.

[29] J. Lehmann, S. Joseph, Biochar for Environmental Management: Science and Technology, first ed. Earthscan, Sterling, 2009.

[30] L.A. Currie, Nomenclature in evaluation of analytical methods including detection and quantification capabilities (IUPAC Recommendations 1995), Anal. Chim. Acta 391 (1999) 105-126. 